\title{
A Scoping Review of Components of Physician-induced Demand for Designing a Conceptual Framework
}

\author{
Marita Mohammadshahi', Shahrooz Yazdani ${ }^{2}$, Alireza Olyaeemanesh ${ }^{3}$, Ali Akbari Sari' ${ }^{1}$, Mehdi Yaseri $^{4}$, \\ Sara Emamgholipour Sefiddashti ${ }^{1}$ \\ ${ }^{1}$ Department of Health Management and Economics, School of Public Health, Tehran University of Medical Sciences, Tehran, Iran; ${ }^{2}$ Department of \\ Cardiology, School of Medicine, Alborz University of Medical Sciences, Karaj, Iran; ${ }^{3}$ National Institute of Health Research, Group of Payment and \\ Financial Resources of the Health System, Tehran University of Medical Sciences, Tehran, Iran; ${ }^{4}$ Department of Epidemiology and Biostatistics, \\ School of Public Health, Tehran University of Medical Sciences, Tehran, Iran
}

Objectives: The current study presents a new conceptual framework for physician-induced demand that comprises several influential components and their interactions.

Methods: This framework was developed on the basis of the conceptual model proposed by Labelle. To identify the components that influenced induced demand and their interactions, a scoping review was conducted (from January 1980 to January 2017). Additionally, an expert panel was formed to formulate and expand the framework.

Results: The developed framework comprises 2 main sets of components. First, the supply side includes 9 components: physicians' incentive for pecuniary profit or meeting their target income, physicians' current income, the physician/population ratio, service price (tariff), payment method, consultation time, type of employment of physicians, observable characteristics of the physician, and type and size of the hospital. Second, the demand side includes 3 components: patients' observable characteristics, patients' non-clinical characteristics, and insurance coverage.

Conclusions: A conceptual framework that can clearly describe interactions between the components that influence induced demand is a critical step in providing a scientific basis for understanding physicians' behavior, particularly in the field of health economics.

Key words: Induced demand, Conceptual framework, Target income, Pecuniary incentives, Scoping review

\section{INTRODUCTION}

Physicians' behaviors play a pivotal role in their provision of medical services and are of particular importance for health

Received: October 13, 2018 Accepted: December 10, 2018

Corresponding author: Sara Emamgholipour Sefiddashti, PhD Department of Health Management and Economics, School of Public Health, Tehran University of Medical Sciences, Poursina Street, Tehran 1417613151, Iran.

E-mail: s-emamgholipour@tums.ac.ir

This is an Open Access article distributed under the terms of the Creative Commons Attribution Non-Commercial License (http://creativecommons.org/licenses/by$\mathrm{nc} / 4.0 / /$ which permits unrestricted non-commercial use, distribution, and reproduction in any medium, provided the original work is properly cited. economics, mainly owing to the fact that physicians can benefit from their role as agents and the information asymmetry between physicians and patients by inducing demand [1]. According to McGuire [2], physician-induced demand (PID) occurs when "a physician influences a patient's demand for care against the physician's interpretation of the best interest of the patient" [2]. A wide spectrum of factors can influence PID and have been widely investigated by researchers. Based on the results of these studies, various and sometimes contradictory models have been developed to characterize physicians' behavior. Some models have used utility theory and profit maximization principles to investigate PID [3-5], whereas others have followed the principles of neoclassical theory by using a 
persuasive advertisement model $[6,7]$. Still others have used econometric models, such as structural equation [8], difference-in-difference $[9,10]$, and Poisson models of the impacts of different factors on physicians' behavior [1]. Various studies have considered different factors. Some have investigated the density of physicians in a specified area as a factor that can motivate PID, while others have emphasized the importance of changes in payment methods or insurance coverage $[4,11$ 14]. In general, previous studies have emphasized 1 or 2 factors that can influence PID, or tried to prove the existence of PID using the above-mentioned factors. However, such investigations may be influenced by bias or error, and they therefore have been criticized by a number of economists $[15,16]$. For example, most studies on supplier-induced demand (SID) have found a direct association between service utilization and the number of physicians, and have pointed out that this kind of increase in service utilization is not necessarily due to PID [17-20]. Although studies have shown the existence of PID, most have not been able to provide strong evidence for the existence of SID. However, some researchers have tried to address this issue. For example, Liao [7] used the Physician Ethics Index to tackle the shortcomings of previous studies, and Grytten and Sørensen [21] proposed a more realistic model for PID in a study of payment methods.

Since the concept of PID is very broad and complex, debates on theories and their impacts continue, and there is no united framework. Labelle et al. [22] developed a conceptual model for the definition of PID that has been endorsed and used by many researchers. The current study tried to provide a more expanded conceptual framework by extending this model and describing the influential factors in greater depth, in order to make the association more transparent and thereby promote a better understanding of the concept of PID.

The conceptual framework proposed in this study, which aims to measure the impact of different factors, is based on the conceptual model developed by Labelle et al. [22]. The Labelle model includes the effectiveness of provided services and of the agency relationship to identify the separate concepts of service utilization that have been expressed in different studies. By asking the 2 following questions, in addition to considering patients' health status, the Labelle model explored the impact of induced services. First, if patients had the same information, would they demand the same services? Second, did the provided services have a positive impact on patients' health? Labelle showed the answers in a matrix (Figure 1) [22].

The model investigated the service provision behavior of physicians from 2 perspectives-agency effectiveness and services effectiveness-and described illustrative cases of different types of PID. With regard to the information asymmetry, the agency effectiveness dimension deals with the scope of agency effectiveness, as well as whether physicians act as perfect agents, rather than the existence of the agency relationship. The second dimension shows the impact of physicians' actions on patients' health status, and reflects the marginal benefit for patients from the provided services. Based on the matrix that was developed (Figure 1) [22], if physicians do not act as perfect agents, cells IVa and IVb show SID that has traditionally had negative connotations - utilization that is ineffective, and thus wasteful (Cell IVa) or even harmful (Cell IVb) to patients, and which informed patients would not have chosen to consume.

Based on this conceptual framework, whether provided services align with available clinical practice guidelines may be one of the best ways to identify induced demand. In fact, this approach may address the weak areas of studies that only use increased service utilization as the main indicator of induced demand. Along with this concept, a series of components that influence induced demand should be described, in order to

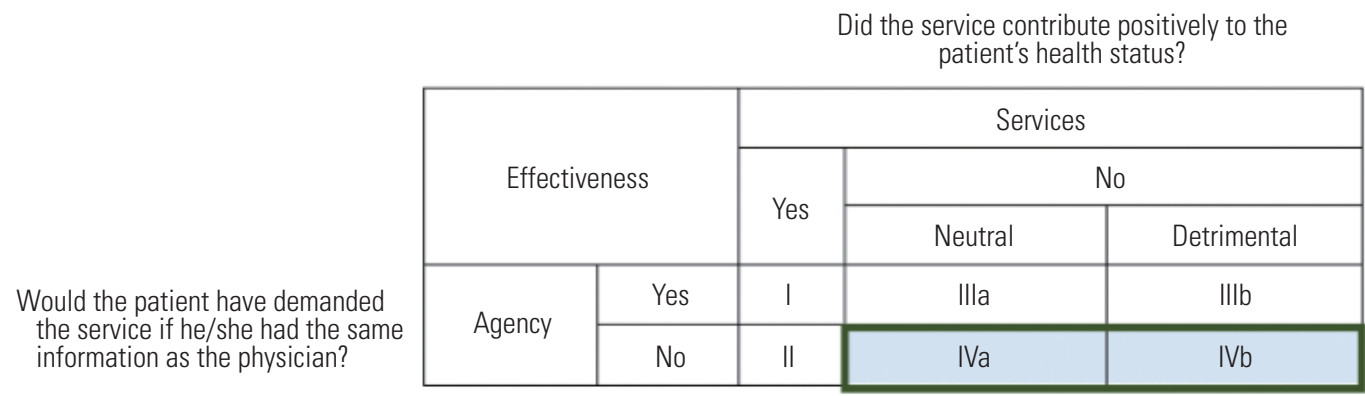

Figure 1. The developed conceptual framework. Source from: Labelle et al. J Health Econ 1994;13(3):347-368 [22], with permission of Elsevier. 
measure their effects and their associations. In today's interactive world, it is undoubtable that the components of induced demand interact with each other and must be considered as a whole in studies. A model based on these components can be used at both micro-levels (e.g., the hospital level) and macrolevels (e.g., the national level).

\section{METHODS}

Since no comprehensive review has been conducted of factors that contribute to PID, a scoping review was conducted to identify the components that influence PID and to understand their interactions. A scoping review is a knowledge synthesis technique that aims to collect and evaluate the current state of knowledge on a particular topic, especially when the issue has not yet been comprehensively reviewed. Thus, the 5 stages of Arksey and O'Malley [23]'s framework for scoping reviews was used, as described below:

\section{Stage 1: Identifying the Initial Research Questions}

The focus of this study was on the components of PID and their relationships, with the goal of designing a conceptual framework, so the initial questions that guided our search were:

(1) What are the components of PID?
(2) How are these components related to each other?

(3) What is the best conceptual framework to show these components and their relationships?

\section{Stage 2: Identifying Relevant Studies}

Key concepts and search terms were developed to identify the literature related to PID. We performed a comprehensive literature search of the major databases, including Scopus, PubMed, Web of Science, ProQuest, the Cochrane Library, and Embase, from January 1, 1980 to January 1, 2017. Moreover, a hand search of the reference lists of selected papers was performed, and Google Scholar was searched to identify any other gray literature. The searches were all done from October 28, through November 3, 2017. For searching the above-mentioned databases, a search strategy appropriate for each database regarding Medical Subject Headings guidelines was employed, with the use of following key words: PID, SID physician density, payment method/income, and service price. The search strategies used to find relevant studies by title and abstract are outlined in Table 1.

\section{Stage 3: Study Selection}

In the initial search, 2154 articles were identified. Through a title review, using the inclusion and exclusion criteria, a large

Table 1. Search strategy of databases

\section{Database}

PubMed

Search (((Supplier induced demand [Title/Abstract]) OR Physician induced demand [Title/Abstract]) OR PID [Title/Abstract]) OR SID [Title/ Abstract]

Search (/(/(Supplier induced demand [Title/Abstract]) OR Physician induced demand [Title/Abstract]) OR PID [Title/Abstract]) OR SID [Title/ Abstract])) AND (payment method OR income) [Title/Abstract]

Search (/((ISupplier induced demand [Title/Abstract]) OR Physician induced demand [Title/Abstract]) OR PID[Title/Abstract]) OR SID [Title/ Abstract])) AND (service price OR tariff) [Title/Abstract]

Search (/(/(Supplier induced demand [Title/Abstract]) OR Physician induced demand [Title/Abstract]) OR PID [Title/Abstract]) OR SID [Title/ Abstract])) AND insurance [Title/Abstract]

Search (U(/(Supplier induced demand [Title/Abstract]) OR Physician induced demand [Title/Abstract]) OR PID [Title/Abstract]) OR SID [Title/ Abstract])) AND physician density [Title/Abstract]

Search (/(/(Supplier induced demand [Title/Abstract]) OR Physician induced demand [Title/Abstract]) OR PID [Title/Abstract]) OR SID [Title/ Abstract])) AND asymmetric information [Title/Abstract]

Cochrane Library \#1 physician Near/2 induced Near/2 demand, \#2 supplier Near/2 induced Near/2 demand, \#3 SID, \#4 PID, \#5 payment, \#6 insurance, \#7 Physician density, \#8 service price, \#9 asymmetric information, \#10 \#1 0R \#2, \#11 \#3 AND \#4, \#12 \#10 OR \#11, \#13 \#12 AND \#5, \#14 \#12 AND \#6, \#15 \#12 AND \#7, \#16 \#12 AND \#8, \#17 \#12 AND \#9

Scopus ((TITLE-ABS-KEY(physician induced demand)) OR (TITLE-ABS-KEY(Supplier induced demand)) OR (TITLE-ABS-KEY (PID)) OR (TITLE-ABS-KEY (SID))) AND (TITLE-ABS-KEY (payment)) OR AND (TITLE-ABS-KEY (insurance)) OR (TITLE-ABS-KEY (physician density)) OR (TITLE-ABS-KEY (service price)) OR (TITLE-ABS-KEY (asymmetric information)) AND (LIMIT-TO (LANGUAGE, "English"))

Embase induced:ab,ti AND demand:ab,ti OR (supplier:ab,ti AND induced:ab,ti AND demand:ab,ti) OR (physician:ab,ti AND induced:ab,ti AND demand:ab,ti) AND payment OR ('density'/exp OR density) OR insurance: ab, ti OR asymmetric information: ab, ti OR service price: ab, ti

Web of Sciences TITLE: (induced NEAR/2 demand) OR TITLE: (physician NEAR/2 induced NEAR/2 demand) OR TITLE: (supplier NEAR/2 induced NEAR/2 demand) AND (TITLE: (payment) OR TITLE: (insurance) OR (physician density) OR (service price) OR (asymmetric information))

ProQuest TITLE: (induced NEAR/2 demand) OR TITLE: (physician NEAR/2 induced NEAR/2 demand) OR TITLE: (supplier NEAR/2 induced NEAR/2 demand) AND (TITLE: (payment) OR TITLE: (insurance) OR (physician density) OR (service price) OR (asymmetric information)) 


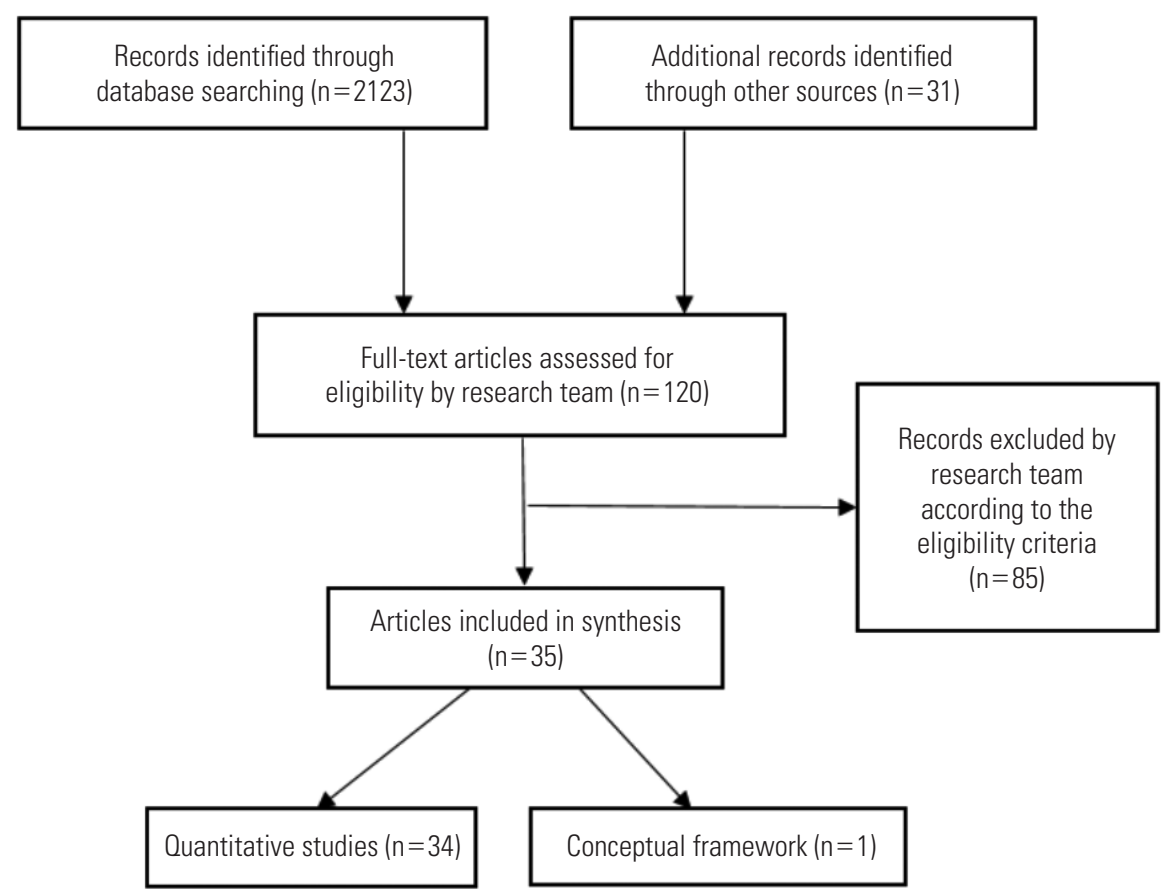

Figure 2. Flow of research through the stage of review.

number of studies that were irrelevant, duplicates, not published in English, or did not have the appropriate timeframe were excluded. For studies to be eligible for this review, they must have presented specific components and the metrics used to measure their relationships. Two reviewers (MM, SES) followed the above-defined criteria separately and screened the studies by title, abstract, author, year of publication, country/region, and article type; they were in agreement for $90 \%$ of the identified literature, and cases of disagreement were resolved through consensus; subsequent, 35 studies remained. Figure 2 shows the study selection process.

\section{Stage 4: Data Charting and Collation}

The authors created a worksheet in Excel, and the author, year, and location of each study, as well as the variables used to assess PID and their relationships were entered into this Excel file to manage the data.

\section{Stage 5: Summary and Reporting of Findings}

We used the narrative method to extract common themes of the components and their relationships, and the results were then summarized. The identified components were divided into 2 main groups - supply-side and demand-sidewhich are explained further in the Results section.

Next, a panel of 10 experts was assembled, and their opin- ions were solicited about the new conceptual framework that was developed by the authors. All the experts had a high level of education and played important roles in decision-making and policy-making about the health care system. They included members of Social Security Insurance, the Ministry of Health and Medical Education, hospital administrators, and professors. The participants were pessimistic about the expanded conceptual framework for PID. In response to their suggestions, some components and the relationships thereof were modified, and the model was finalized.

\section{RESULTS}

As shown in Figure 2, 2154 records were identified in the initial search. From these results, the full text of 120 potentially relevant studies was assessed by the research team, and 85 studies were excluded because they did not meet the eligibility criteria. Of the remaining studies, 34 studies were quantitative, and 1 study was about a conceptual framework. The list of the studies is available in Table S1.

The studies involved a broad range of factors and were highly heterogeneous according to the applied theoretical frameworks and methodology. Therefore, we present a brief discussion of the components and their relationships. The components were classified into 2 main categories-supply- 
side with 9 components and demand-side with 3 components - that are presented in more detail below.

\section{Supply-side Components}

\section{Physicians' incentive for pecuniary profit (or to meet their target income)}

From a mercantilist perspective, the correctness of a behavior or action should be judged by measuring or evaluating its outcomes, and an action or behavior is deemed to be correct if it has better outcomes and is more worthwhile [24]. In a competitive medical market, particularly for general practitioners, achieving a target income (an income that the person thinks he or she deserves) provides a strong incentive for increasing activity and providing services that result in more profit. Induced demand is a way to fill the gap between current and target income, up to the point that the disutility that arises from induced demand becomes equal to the utility of the higher income. Therefore, personal benefit is a major incentive for inducing demand, and various studies have considered this through different lenses, such as medical ethics indicators $[6,7,25]$.

\section{Physicians' income}

As described in the general theory of employment, interest, and money proposed by Keynesian models, "nominal wages are sticky-down" - that is, individuals oppose reductions in their income [26]. Physicians, although their incomes are higher than those of many other occupations, are not an exception to this generalization, and respond to each factor that results in a reduction of their current income. Since physicians are patients' agents and can manipulate their demand, when they encounter reduced income, they can compensate in various ways (e.g., increasing the quantity of provided services, changing the combination of provided services, or increasing self-referrals and consultation length) [27-29].

\section{Physician/population ratio}

The physician/population ratio is an important factor underlying induced demand. In health markets, when prices are fixed, an increase in the number of physicians reduces the market share of each provider and creates unwanted nonprice competition [2]. Studies of physicians' geographical distribution have shown that higher demand, compared to supply, has a greater influence on their income. Therefore, to maintain their previous income levels, they induce demand, although it should be noted that in this case, the increased utilization is not related to the increased accessibility to healthcare services $[2,7]$.

\section{Price of services}

Changes in the price of services result in variations in the price/cost ratio, which directly motivate physicians who intend to maximize their profit to induce demand. When governments reduce the prices to balance the market, or keep prices within a predetermined threshold, the substitution effect increases utilization. Such price changes also create an income effect. Various studies have investigated the impact of price changes, and most have shown that reduced prices, which in turn result in lower incomes, motivate physicians to induce demand [30,31].

\section{Payment methods}

Payment methods have complex impacts on physicians' behavior and induced demand. Physicians can be compensated either directly through out-of-pocket payments or indirectly by a third party. Various countries use different payment methods, and it has been shown that fee-for-services and perdiem payments can be used by physicians to manipulate the volume or intensity of provided services more easily, thereby allowing them to increase their income and simultaneously influence the quality of provided services. Furthermore, under per capita arrangements, physicians can increase the number of enlisted patients to increase or maintain previous levels of income and at the same time increase the frequency of followup visits [32-34].

\section{Consultation time per visit or service}

In many countries, governments have set minimum standards for the length of consultations, and depending on the payment method, physicians receive compensation that consists of 2 elements: a fixed part and a variable part. In such a system, if a consultation lasts longer than a predetermined threshold, the insurer or patient should pay an extra amount. This type of payment can provide incentives for physicians to induce demand by increasing the duration of consultations, particularly when their income declines (e.g., due to an increased number of physicians or a decreased number of patients) $[13,21,35]$. 


\section{Type of recruitment of physicians}

Physicians in different countries work under different employment arrangements. Some are employed by the government, or have a contract with the government, while other work privately. Under the first type of arrangement, they receive a fixed and predetermined salary, and therefore incentives to induce demand are almost removed. In the latter circumstances, the type of contract is a strong determinant of induced demand incentives. Under independent, private arrangements where patients compensate physicians for provided services, physicians can easily provide unnecessary services $[7,21,35]$.

\section{Visible characteristics of the physician}

This component comprises a set of characteristics that are visible to researchers, including marital status, sex, age, duration of clinical experience, level of training, and medical specialty. A large number of studies have investigated the impact of these factors on incentives for inducing demand. They showed that physicians with different visible features assigned different weights to their income, leisure and work preferences, professional reputation, and motivation to provide unnecessary services $[21,33,36]$.

\section{Type and size of hospitals}

The number of beds or annual average number of patients determines the size of a hospital. Studies have shown that hospital size influences overutilization, owing to financial pressures and variation in the number of patients across hospitals. Usually in larger hospitals, fixed costs are proportionally higher, which creates incentives for providing more expensive services to increase the return on investment. This is more common in private hospitals than in public ones $[8,10]$.

\section{Demand-side Components \\ Patients' visible clinical features}

This component refers to patients' health status, and it is categorized based on diagnosis-related group. Visible characteristics can be observed by physicians, thereby providing them with an information advantage that can be used for personal benefit. The patient's medical status can vary from minor to highly complex. Overall, physicians are less likely to induce demand if a patient's illness is minor, because the level of the information asymmetry will not be as significant, but in patients with highly-complex medical conditions, this gap is larger, meaning that depending on the physician's interest, the volume of services can be manipulated $[1,8]$.

\section{Patients' visible non-clinical features}

Some parameters within this component, such as socioeconomic status (education level, occupation, marital status, and residence area), enable physicians to assess the level of medical information available to patients and their welfare. Furthermore, some of these demographic features, such as age and sex, are used to assess patients' health. Studies have shown that individuals with lower socioeconomic status are more likely to have limited medical information, making it easier to manipulate their demand for physicians $[8,33]$.

\section{Insurance coverage}

Health systems use various methods to finance healthcare services. In some health systems, patients directly pay the costs, while in other systems, insurers pay the bills. In addition, there are a number of systems that use mixed methods. Insurance coverage and its types play an important role in induced demand, because on the one hand, the payment method is determined by insurers, and on the other hand, physicians can readily provide extra services $[33,37,38]$.

\section{DISCUSSION}

In the current study, a model of induced demand and its related components was developed based on the framework proposed by Labelle et al. [22]. In addition, influential components were proposed according to a scoping review, and their relationships were described based on the results of an expert panel. After reaching consensus, an integrated conceptual framework was proposed (Figure 3).

As discussed in most of the literature on the topic, induced demand originates from physicians' desire to acquire financial benefits. In most previous studies, PID has been considered within the context of medical ethics by measuring physicians' and patients' utility, which directly influence induced demand $[6,7]$. Physicians' income is another important factor that directly influences their utility. Therefore, any factor that reduces physicians' income would result in decreased utility, which in turn provides incentives to compensate for this reduction via inducing demand. Therefore, based on the components discussed above, income influences induced demand through a desire to gain more financial benefits $[4,6,39]$. 


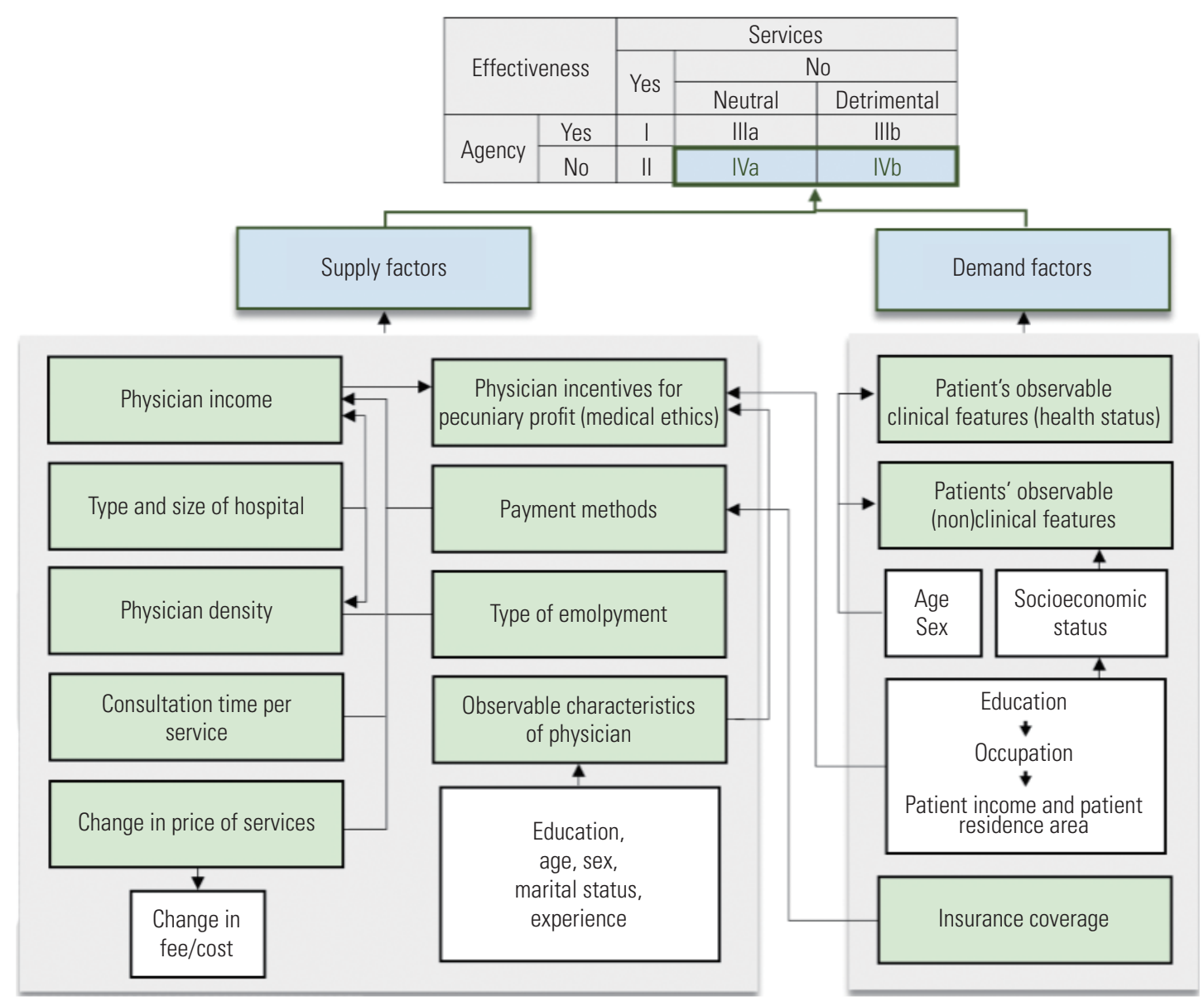

Figure 3. Conceptual framework of physician induced demand.

However, if the equilibrium price or tariff is considered constant at a certain level and the supply of physicians increases in response to demand for services (i.e., there is no accessibility effect), physicians' income will be affected. Feldman and Sloan [27] showed that physicians' density did not have a direct impact on induced demand, and suggested that income was a mediating factor. Thus, physicians' density and induced demand show an indirect association. In addition, if governments change the reimbursements in a manner that is inconsistent with physicians' desires, physicians can react in a way that involves both income and substitution effects. When prices decline, the price/cost ratio will decline, which results in lower incomes for physicians, in turn leading to induced demand $[40,41]$. Payment methods that link output to income provide an incentive to induce demand to increase income. If physicians show income-maximizing behavior, they seek to acquire the maximum possible income from the services that they provide. Unless the marginal cost is higher than the marginal income, physicians can maximize their income by pro- viding additional services. Consequently, this component influences induced demand through income [32,41].

The allotted time per patient and the type of physician recruitment, which are associated with the payment method, indirectly influence induced demand through income. As Grytten and Sørensen [21] and van Dijk et al. [35] noted, when income declines, physicians who work privately or on a contract basis are motivated to induce demand. Visible characteristics determine the behavior and altruism of physicians. In other words, factors that are categorized as part of this component can influence the profit-seeking behavior of physicians. For this reason, many studies have considered this component as an explanatory vector $[7,29]$.

In analyses conducted at the hospital level, the type and size of the hospital are important factors that indirectly influence induced demand, due to their impact on physicians' income. Since a part of a physician's income is determined on a per capita basis, if the number of patients treated at a hospital declines, their income also declines. Therefore, because increas- 
ing the number of hospitalized patients is an option to decrease financial pressures and costs, stakeholders could motivate physicians to induce demand. However, the number of physicians working at a hospital is related to the physician/patient ratio, which impacts their per capita reimbursement. In other words, the more physicians, the less role per capita reimbursement will play $[8,9]$.

So far, we have discussed supply-side components, but there are also 3 demand-side components. The first is related to visible clinical features, which can only be observed by physicians. Since these characteristics determine the health status of patients, based on the Labelle model, they are beneficial for identifying the effectiveness of provided services, as physicians determine the appropriateness of a service based on these characteristics [22]. Visible non-clinical characteristics of patients (i.e., age, sex, education, marital status and residence area) are also among the factors that physicians consider when determining whether to provide extra services; as discussed above, this component reflects patients' level of clinical information [8]. Regarding the component of patients' insurance coverage, on the one hand, insurance coverage determines how physicians are paid, and on the other hand, when patients are insured, physicians can induce demand more easily because a third party pays the charge and the patients' out-of-pocket expenses are low. Therefore, this component acts on both the demand and supply sides $[8,37]$.

\section{Limitations}

Since this scoping review had a broad search scope and the volume of results was quite large, more time was needed to screen articles than is the case for other types of review studies. In addition, there may be additional components that were not mentioned in the present framework, because the findings of this study were based on a review of the existing literature.

\section{CONCLUSION}

It is becoming evident that PID is a multilevel issue with different components that affect each other, so this scoping review was useful for recognizing all aspects of this field, better understanding the issues, and identifying gaps in the existing literature.

Describing a conceptual framework for induced demand, as a basis for identifying its components and their relationships, motivates researchers to investigate and test these related components. Testing a hypothesis on induced demand by considering 1 or 2 components likely leads to bias, because of the wide scope of induced demand and the many factors that contribute to it. In addition, a multilevel investigation of the health sector would seem to be needed to investigate induced demand. A multilevel structural equation model may be among the most useful models for investigating induced demand, because it shows both casual relationships and the multilevel structure of health systems.

Researchers in the health sector are trying to develop a comprehensive model for induced demand. Although many studies have investigated these relationships, previously-developed models fail to show the broadness and links between components, and these investigations have been conducted in the absence of a conceptual framework. With the groundbreaking advances of science in the present century, particularly in the field of econometrics, it is expected that researchers will do their best to develop a comprehensive, universallyapproved conceptual framework.

The conceptual framework developed in the current study attempts to show the complexity of the relationships among different components to provide a broader scientific vision for health economics. Since no unified theory exists regarding induced demand, the authors hope that the current model can provide a basis for consensus and brainstorming ideas to identify strategies for reform, because induced demand is related both to patients' health and to the efficient use of resources.

\section{SUPPLEMENTARY MATERIALS}

Supplementary material is available at https://www.jpmph. org/

\section{CONFLICT OF INTEREST}

The authors have no conflicts of interest associated with the material presented in this paper.

\section{ACKNOWLEDGEMENTS}

The authors acknowledge all relevant academic and administrative entities, especially the Clinical Research Development Unit of Shahid Rajaei Hospital, Alborz University of Medical Sciences (ABZUMS), which helped us by providing useful comments for finalizing the manuscript. This project was conduct- 
ed with the financial support of Tehran University of Medical Sciences (TUMS) (grant No. 2419676).

\section{ORCID}

Marita Mohammadshahi https://orcid.org/0000-00026956-0327

Shahrooz Yazdani https://orcid.org/0000-0002-8637-8439

Alireza Olyaeemanesh http://www.orcid.org/0000-00027417-2417

Ali Akbari Sari http://www.orcid.org/0000-0001-7755-7665 Mehdi Yaseri http://www.orcid.org/0000-0002-4066-873X

Sara Emamgholipour Sefiddashti http://orcid.org/00000001-8654-6554

\section{REFERENCES}

1. Amporfu E. Private hospital accreditation and inducement of care under the ghanaian national insurance scheme. Health Econ Rev 2011;1(1):13.

2. McGuire TG. Physician agency and payment for primary medical care. In: Sherry Glied S, Smith PC, editors. The Oxford handbook of health economics. Oxford: Oxford University Press; 2011, p. 503-517.

3. Folland S, Goodman AC, Stano M. The economics of health and health care. 8th ed. New York: Routledge; 2017, p. 195-207.

4. Munkerud SF. Decision-making in general practice: the effect of financial incentives on the use of laboratory analyses. Eur J Health Econ 2012;13(2):169-180.

5. Iversen $\mathrm{T}$. The effects of a patient shortage on general practitioners' future income and list of patients. J Health Econ 2004; 23(4):673-694.

6. De Jaegher $K$, Jegers M. A model of physician behaviour with demand inducement. J Health Econ 2000;19(2):231-258.

7. Liao CC. Evidence of physician (supplier) induced demand and physician altruism [dissertation]. Detroit: Wayne State University; 2009.

8. Arrieta A. Over-utilization of cesarean sections and misclassification error. Health Serv Outcomes Res Methodol 2015;15(1): 54-67.

9. Shigeoka H, Fushimi K. Supplier-induced demand for newborn treatment: evidence from Japan. J Health Econ 2014;35:162178.

10. Ma KZ. Fertility rate, use of cesarean delivery, and the role of information gap: evidence from Taiwan [dissertation]. Chapel
Hill: University of North Carolina; 2007.

11. Xirasagar S, Lin HC. Physician supply, supplier-induced demand and competition: empirical evidence from a single-payer system. Int J Health Plann Manage 2006;21(2):117-131.

12. Busato A, Künzi B. Primary care physician supply and other key determinants of health care utilisation: the case of Switzerland. BMC Health Serv Res 2008;8:8.

13. Grytten J, Carlsen F, Skau I. Primary physicians' response to changes in fees. Eur J Health Econ 2008;9(2):117-125.

14. Dai T. Incentives in U.S. healthcare operations. Decis Sci 2015; 46(2):455-463.

15. Mooney G. Challenging health economics. Oxford: Oxford University Press; 2009, p. 35-49.

16. Garber AM, Sox HC. The U.S. physician workforce: serious questions raised, answers needed. Ann Intern Med 2004;141(9): 732-734.

17. Wilensky GR, Rossiter LF. The relative importance of physicianinduced demand in the demand for medical care. Milbank Mem Fund Q Health Soc 1983;61(2):252-277.

18. Welch WP, Miller ME, Welch HG, Fisher ES, Wennberg JE. Geographic variation in expenditures for physicians' services in the United States. N Engl J Med 1993;328(9):621-627.

19. Hemenway D, Fallon D. Testing for physician-induced demand with hypothetical cases. Med Care 1985;23(4):344-349.

20. Grytten J. Supplier inducement--its relative effect on demand and utilization. Community Dent Oral Epidemiol 1992;20(1): 6-9.

21. Grytten J, Sørensen R. Type of contract and supplier-induced demand for primary physicians in Norway. J Health Econ 2001; 20(3):379-393.

22. Labelle R, Stoddart G, Rice T. A re-examination of the meaning and importance of supplier-induced demand. J Health Econ 1994;13(3):347-368.

23. Arksey H, O'Malley L. Scoping studies: towards a methodological framework. Int J Soc Res Methodol 2005;8(1):19-32.

24. Bentham J. An introduction to the principles of morals and legislation; 2017 [cited 2018 Dec 20]. Available from: https:// www.earlymoderntexts.com/assets/pdfs/bentham1780.pdf.

25. Tussing AD, Wojtowycz MA. Physician-induced demand by Irish GPs. Soc Sci Med 1986;23(9):851-860.

26. Chari VV, Kehoe PJ, McGrattan ER. New Keynesian models: not yet useful for policy analysis. Am Econ J Macroecon 2009;1(1): 242-266.

27. Feldman R, Sloan F. Competition among physicians, revisited. J Health Polit Policy Law 1988;13(2):239-261. 
28. Sekimoto M, li M. Supplier-induced demand for chronic disease care in Japan: multilevel analysis of the association between physician density and physician-patient encounter frequency. Value Health Reg Issues 2015;6:103-110.

29. Busato A, Matter $P$, Künzi B. Factors related to treatment intensity in Swiss primary care. BMC Health Serv Res 2009;9:49.

30. Rochaix L. Information asymmetry and search in the market for physicians' services. J Health Econ 1989;8(1):53-84.

31. Delattre E, Dormont B. Fixed fees and physician-induced demand: a panel data study on French physicians. Health Econ 2003;12(9):741-754.

32. Mathes T, Pieper D, Mosch CG, Jaschinski T, Eikermann M. Payment methods for hospitals. Cochrane Database of Syst Rev 2014;6:CD011156.

33. McKinlay JB, Potter DA, Feldman HA. Non-medical influences on medical decision-making. Soc Sci Med 1996;42(5):769-776.

34. Hadley J, Reschovsky JD. Medicare fees and physicians' medicare service volume: beneficiaries treated and services per beneficiary. Int J Health Care Finance Econ 2006;6(2):131-150.

35. van Dijk CE, Verheij RA, te Brake H, Spreeuwenberg P, Groenewegen PP, de Bakker DH. Changes in the remuneration sys- tem for general practitioners: effects on contact type and consultation length. Eur J Health Econ 2014;15(1):83-91.

36. Kinder K. Assessing the impact of payment method and practice setting on German physicians' practice patterns. J Ambul Care Manage 2001;24(2):11-18.

37. van Dijk CE, van den Berg B, Verheij RA, Spreeuwenberg P, Groenewegen PP, de Bakker DH. Moral hazard and supplierinduced demand: empirical evidence in general practice. Health Econ 2013;22(3):340-352.

38. Kim B. Do doctors induce demand? Pac Econ Rev 2010;15(4): 554-575

39. Rice TH. The impact of changing medicare reimbursement rates on physician-induced demand. Med Care 1983;21(8): 803-815.

40. Carlsen F, Grytten J. Consumer satisfaction and supplier induced demand. J Health Econ 2000;19(5):731-753.

41. Gosden T, Forland F, Kristiansen IS, Sutton M, Leese B, Giuffrida $A$, et al. Impact of payment method on behaviour of primary care physicians: a systematic review. J Health Serv Res Policy 2001;6(1):44-55. 